\title{
$Q$-operators for higher spin eight vertex models \\ with a rational anisotropy parameter
}

\author{
Takashi TAKEBE* \\ Faculty of Mathematics, \\ International Laboratory of Representation Theory \\ and Mathematical Physics \\ National Research University Higher School of Economics \\ Usacheva Street 6, Moscow, 119048, Russia.
}

\begin{abstract}
$Q$-operators for generalised eight vertex models associated to higher spin representations of the Sklyanin algebra are constructed by Baxter's first method and Fabricius's method, when the anisotropy parameter is rational.
\end{abstract}

\section{Introduction}

This paper is a continuation of [T4], in which we constructed a $Q$-operator of the highger spin generalisation of the eight vertex model. In the present paper we construct the $Q$-operator of the same model with a rational anisotropy parameter $\eta$, following Baxter's 1972 paper [B1] and Fabricius's paper [F].

Baxter [B1] introduced the $Q$-operator as an auxiliary tool to find eigenvalues of the transfer matrix of the eight vertex model. Its fundamental property is the $T Q$-relation,

$$
T(u) Q(u)=Q(u) T(u)=h_{-}(u) Q(u-2 \eta)+h_{+}(u) Q(u+2 \eta),
$$

with the transfer matrix $T(u)$, the commutativity with itself, $\left[Q(u), Q\left(u^{\prime}\right)\right]=$ 0 , and the quasi-periodicity with respect to the shift of the spectral parameter $u \mapsto u+1, u \mapsto u+\tau$.

*e-mail: ttakebe@hse.ru 
We constructed such an operator for the higher spin eight vertex models in [T4], following Baxter's 1973 paper [B2] and his book [B3], when the number of sites $N$ was even. This model is defined by spin $l$ representations of the Sklyanin algebra [S1], [S2] and has been studied by the author in [T1], [T2], [T3] by means of the generalised algebraic Bethe Ansatz of Takhtajan and Faddeev [TF]. The results of [T4] not only confirmed the results obtained by the algebraic Bethe Ansatz in [T1], but also proved the sum rule of Behte roots conjectured in [T2].

In the present paper we show that Baxter's very first construction of the $Q$-operator in [B1] and Fabricius's new construction in [F] (see also [Roa2]) can be applied to the higher spin model with a rational anisotropy parameter. Recall that in [T1] and [T2] the generalised Bethe Ansatz was shown to be applicable to the higher spin model under the assumption, $N l \in \mathbb{Z}$, but our construction of the $Q$-operator in [T4] was valid only for even $N$. In particular, the case when the number of sites $N$ is odd and the spin $l$ is an integer was excluded in [T4, while the generalised Bethe Ansatz was applied in [T1] and [T2 to such a case as well. We show that Baxter's construction in [B1] gives the $Q$-operator also in this case. Thus we fill the gap between the generalised Bethe Ansatz and the $Q$-operator approach for the higher spin model.

As was pointed out by Fabricius and McCoy in [FM1, Baxter's 1972 construction has a weak point. In B1 the $Q$-operator $Q(u)$ was defined by $Q(u)=Q_{R}(u) Q_{R}\left(u_{0}\right)^{-1}$, where $Q_{R}(u)$ is an intermediate object in the construction and $u_{0}$ is a point, at which $Q_{R}(u)$ is invertible. Fabricius and McCoy found numerically that $Q_{R}(u)$ might be degenerate for certain $\eta$, which means that this construction cannot be applied.

We do not touch upon this problem and assume that $Q_{R}(u)$ is nondegenerate at generic $u$. The reason is not because we think this problem negligible, but, on the contrary, because it requires serious study, to which another article should be devoted. Anyway, according to [FM2], either Baxter's or Fabricius's construction works for any rational anisotropy parameter 1 .

What is remarkable in our construction is that Baxter's and Fabricius's methods work almost just as they are, although they seem to make use of special form of matrix elements.

This paper is organised as follows: in 92 we recall the model introduced in [T1] by defining its transfer matrix and general strategy of the construction

\footnotetext{
${ }^{1}$ The normalisation of theta functions by Fabricius and McCoy in FM1, FM2, FM3 and that by Sklyanin in $\mathbf{S 2}$ differs by a modular transformation. (See the explicit form of the $R$-matrix, A.5 in this paper.) Since we use Sklyanin's normalisation, our cases correspond to $m_{1}=0$ in FM1.
} 
of the $Q$-operator, which is essentially due to Baxter [B1]: First we construct an operator $Q_{R}(u)$ which satisfies the $T Q$-relation (2.5) as the trace of a product of auxiliary operators $S(u)$. Then by transposing $Q_{R}(u)$ (in our case, by taking the Hermitian adjoint with respect to the Sklyanin form) we obtain an operator $Q_{L}(u)$ satisfying the $Q T$-relation (2.6). Operators $Q_{R}(u)$ and $Q_{L}(u)$ satisfy the commutation relation (3.24), from which follows the definition of the $Q$-operator, (3.31).

In $\$ 3$ we construct a $Q$-operator, following Baxter's 1972 paper [B1], and in $\S 4$ we follow essentially Fabricius's paper [F]. The auxiliary operatorvalued matrix $S(u)$ in $\oint 3$ is tridiagonal in ordinary sense (3.1) and $S(u)$ in $\$ 4$ is a tridiagonal matrix as a matrix with cyclic indices (4.1).

In $\$ 4$ we made a slight and technical modification to Fabricius's construction by using the results in our old work [T1], [T2]. This modification gives an interpretation of Baxter's strategy in [B1] from the viewpoint of the generalised algebraic Bethe Ansatz by Takhtajan and Faddeev [TF]: Baxter used a "half" of the Gauss decomposition of the gauge transfromation matrix in the generalised algebraic Bethe Ansatz. See Remark 4.1.

Sklyanin's unitary operators $U_{1}$ and $U_{3}$ commute with $Q$-operators constructed in $\$ 3$ and $\$ 4$ up to shifts of the spectral parameter and a constant multiplication, as is proved in $\$ 3.4$ and $\$ 4.3$.

We make several concluding remarks with comments on related works in the final section $\$ 5$.

All the necessary facts about the Sklyanin algebra are collected in Appendix A. In the proof of the commutation relation of $Q_{R}$ and $Q_{L}$ we need to compute the Sklyanin form (a scalar product in the space of theta functions) of several vectors, using Rosengren's results [Ros1] and [Ros2]. We summarise necessary results in Appendix B.

\section{Notations}

Throughout this paper we use the following notations and symbols.

- $N \in \mathbb{Z}_{>0}$ : the number of sites.

- $l \in \frac{1}{2} \mathbb{Z}_{>0}$ : the spin of the representation at each site. We assume that $N l \in \mathbb{Z}$. Namely, if $l$ is a half integer, then $N$ should be even.

- $\tau \in i \mathbb{R}_{>0}$; the elliptic modulus, which is purely imaginary.

- $\eta=\frac{r^{\prime}}{2 l r} \in\left[-\frac{1}{2(2 l+1)}, \frac{1}{2(2 l+1)}\right]\left(r, r^{\prime} \in \mathbb{Z}, r>0\right)$ : the anisotropy parameter. 
- The notations for the theta functions are the same as those in Mumford's book [Mu] (cf. Sklyanin's papers [S1], S2]):

$$
\theta_{a b}(z, \tau)=\sum_{n \in \mathbb{Z}} \exp \left(\pi i\left(\frac{a}{2}+n\right)^{2} \tau+2 \pi i\left(\frac{a}{2}+n\right)\left(\frac{b}{2}+z\right)\right)
$$

(cf. Jacobi's notation (e.g., [WW]): $\vartheta_{1}(\pi z, \tau)=-\theta_{11}(z, \tau), \vartheta_{2}(\pi z, \tau)=$ $\theta_{10}(z, \tau), \vartheta_{3}(\pi z, \tau)=\theta_{00}(z, \tau), \vartheta_{4}(\pi z, \tau)=\theta_{01}(z, \tau)$.)

- We denote $\theta_{11}(z, \tau)$ by $[z]$ for simplicity.

- $[z]_{k}:=\prod_{j=0}^{k-1}[z+2 j \eta]=[z][z+2 \eta] \cdots[z+2(k-1) \eta]$ for $k=1,2, \ldots$, $[z]_{0}=1$. The following function is important.

$$
[z ; a]_{k}:=[z+a]_{k}[-z+a]_{k} .
$$

- The Pauli matrices are defined as usual:

$$
\sigma^{0}=\left(\begin{array}{ll}
1 & 0 \\
0 & 1
\end{array}\right), \quad \sigma^{1}=\left(\begin{array}{ll}
0 & 1 \\
1 & 0
\end{array}\right), \quad \sigma^{2}=\left(\begin{array}{cc}
0 & -i \\
i & 0
\end{array}\right), \quad \sigma^{3}=\left(\begin{array}{cc}
1 & 0 \\
0 & -1
\end{array}\right) .
$$

\section{Definition of the model and the $Q$-operator}

In this section we review the higher spin generalisation of the eight vertex model, which was studied in [T1], T2], TT4]. Normalisations are the same as in [T4. For notations see Appendix A.

The local state spaces are the spin $l$ representation space of the Sklyanin algebra $\left(l \in \frac{1}{2} \mathbb{Z}_{>0}\right) V_{i}(i=1, \ldots, N): V_{i} \cong \Theta_{00}^{4 l+}$. The total Hilbert space $\mathcal{H}$ is their tensor product:

$$
\mathcal{H}:=V_{N} \otimes \cdots \otimes V_{1},
$$

while the auxiliary space $V_{0}$ is a two-dimensional space: $V_{0} \cong \mathbb{C}^{2}$.

The transfer matrix $T(u)$ of the model is defined by

$$
T(u):=\operatorname{tr}_{0} L_{N}(u) L_{N-1}(u) \cdots L_{1}(u),
$$

where $\operatorname{tr}_{0}$ is the trace over the space $V_{0}$ and the $L$-operator $L_{n}(u) \in \operatorname{End}_{\mathbb{C}}(\mathcal{H} \otimes$ $\left.V_{0}\right)$ is defined by a representation of the Sklyanin algebra on $\mathcal{H}, \rho_{n}:=1 \otimes$ $\cdots \otimes \rho^{n-\text { th component }} \otimes \cdots \otimes 1$, as

$$
L_{n}(u)=\sum_{a=0}^{3} W_{a}^{L}(u) \rho_{n}\left(S^{a}\right) \otimes \sigma^{a} .
$$


When the spin $l$ is $1 / 2$, the transfer matrix $T(u)$ is essentially that of the eight vertex model. As usual, the $R L L$-relation (A.1) leads to the commutativity of the transfer matrix:

$$
T(u) T\left(u^{\prime}\right)=T\left(u^{\prime}\right) T(u)
$$

As in the previous paper [T4], our goal in this article is to construct a $Q$-operator $Q(u)$, an operator satisfying the commutation relations:

$$
\begin{aligned}
& T(u) Q(u)=h_{-}(u) Q(u-2 \eta)+h_{+}(u) Q(u+2 \eta), \\
& Q(u) T(u)=h_{-}(u) Q(u-2 \eta)+h_{+}(u) Q(u+2 \eta), \\
& Q(u) Q(v)=Q(v) Q(u),
\end{aligned}
$$

where the functions $h_{ \pm}(u)$ are defined by

$$
h_{ \pm}(u):=(2[u \mp 2 l \eta])^{N} .
$$

We apply two methods to this model, one by Baxter [B1] and the other by Fabricius [F] (see also [Roa2]). Their main strategies are essentially the same, which is due to Baxter [B1].

1. First we construct the " $Q_{R^{-}}$operator" of the form

$$
Q_{R}(u)=\operatorname{tr}_{\mathbb{C}^{r}} S_{N}(u) S_{N-1}(u) \cdots S_{1}(u),
$$

satisfying the $T Q$-relation (2.5) $)$. The operator $S_{n}(u) \in \operatorname{Hom}_{\mathbb{C}}\left(\left(\mathbb{C}^{2 l+1}\right)^{\otimes N} \otimes\right.$ $\left.\mathbb{C}^{r_{0}}, \mathcal{H} \otimes \mathbb{C}^{r_{0}}\right)$ is an $r_{0} \times r_{0}$-matrix of linear maps $\left(\mathbb{C}^{2 l+1}\right)^{\otimes N} \rightarrow \mathcal{H}$ :

$$
S_{n}(u)=\left(\left(S_{j^{\prime \prime}}^{i^{\prime \prime}}(u)\right)_{n}\right)_{i^{\prime \prime}, j^{\prime \prime}=1, \ldots, r_{0}},
$$

where

$$
\left(S_{j^{\prime \prime}}^{i^{\prime \prime}}(u)\right)_{n}=\operatorname{Id}_{V_{N}} \otimes \cdots \otimes \stackrel{S_{j^{\prime \prime}}^{i^{\prime \prime}}(u)}{n-\text {-th }} \otimes \cdots \otimes \operatorname{Id}_{V_{1}}
$$

and $S_{j^{\prime \prime}}^{i^{\prime \prime}}(u): \mathbb{C}^{2 l+1} \rightarrow \Theta_{00}^{4 l+}$. We denote

$$
S(u)=\left(S_{j^{\prime \prime}}^{i^{\prime \prime}}(u)\right)_{i^{\prime \prime}, j^{\prime \prime}=1, \ldots, r_{0}} .
$$

The matrix size $r_{0}$ for the first method is $r$, while $r_{0}=2 r$ for the second method. (Recall that $r$ is a factor of the denominator of $\eta=\frac{r^{\prime}}{2 l r}$.) 
2. The product $T(u) Q_{R}(u)$ has the form

$$
T(u) Q_{R}(u)=\operatorname{tr}_{V_{0} \otimes \mathbb{C}^{r}} \prod_{1 \leq n \leq N}^{\curvearrowleft}\left(L_{n}(u) \otimes S_{n}(u)\right),
$$

where the tensor product of $L_{n}(u)$ and $S_{n}(u)$ is a $\left(2 r_{0} \times 2 r_{0}\right)$-matrix with $\operatorname{Hom}_{\mathbb{C}}\left(\left(\mathbb{C}^{2 l+1}\right)^{\otimes N}, \mathcal{H}\right)$-valued elements, $L_{j^{\prime}}^{i^{\prime}}(u) S_{j^{\prime \prime}}^{i^{\prime \prime}}(u)$. We index the components of $V_{0} \cong \mathbb{C}^{2}$ by $i^{\prime}, j^{\prime}= \pm$ and $\mathbb{C}^{r_{0}}$ by $i^{\prime \prime}, j^{\prime \prime} \in\left\{1, \ldots, r_{0}\right\}$.

If there exists a $\left(2 r_{0} \times 2 r_{0}\right)$-matrix $M$ with scalar elements, such that

$$
M^{-1}(L(u) \otimes S(u)) M=\left(\begin{array}{cc}
A(u) & 0 \\
* & D(u)
\end{array}\right) \text { or }\left(\begin{array}{cc}
A(u) & * \\
0 & D(u)
\end{array}\right)
$$

then we have

$$
T(u) Q_{R}(u)=\operatorname{tr}_{\mathbb{C}^{r_{0}}} A_{N}(u) \cdots A_{1}(u)+\operatorname{tr}_{\mathbb{C}^{r_{0}}} D_{N}(u) \cdots D_{1}(u)
$$

We derive the $T Q$-relation (2.5) from (2.13) by using explicit forms of $A(u)$ and $D(u)$.

3. The $Q_{L}$-operator which satisfies the $Q T$-relation (2.6) is obtained as an adjoint to $Q_{R}$ with a change of the spectral parameter.

4. The commutativity $Q_{L}(u) Q_{R}\left(u^{\prime}\right)=Q_{L}\left(u^{\prime}\right) Q_{R}(u)$ is shown by computing the matrix elements of its both sides explicitly.

5. The $Q$-operator is defined by $Q(u)=Q_{R}(u) Q_{R}\left(u_{0}\right)^{-1}=Q_{L}\left(u_{0}\right)^{-1} Q_{L}(u)$, where $u_{0}$ is chosen so that $Q_{R}\left(u_{0}\right)$ and $Q_{L}\left(u_{0}\right)$ are invertible. The existence of such $u_{0}$ will not be discussed in this paper. (For the eight vertex model, detailed study of degeneracy of $Q_{R}(u)$ is in [FM1].)

The commutation relations (2.5), (2.6) and (2.7) follow automatically from (2.5) for $Q_{R}$, (2.6) for $Q_{L}(u)$ and $Q_{L}(u) Q_{R}\left(u^{\prime}\right)=Q_{L}\left(u^{\prime}\right) Q_{R}(u)$.

6. The $Q$-operator is quasi-periodic in the spectral parameter $u$. The shift of $u$ is equivalent to multipication with involutions $U_{1}^{\otimes N}$ and $U_{3}^{\otimes N}$ on the Hilbert space $\mathcal{H}$.

\section{The $Q$-operator à la Baxter (1972)}

In this section we construct the $Q$-operator for the higher spin eight vertex model, following the strategy sketched in $\$ 2$ with $r_{0}=r$. In this construction, 
the matrix $S(u)(2.11)$ is of the form,

$$
S(u)=\left(\begin{array}{ccccc}
S_{1}^{1}(u) & S_{2}^{1}(u) & & & \\
S_{1}^{2}(u) & 0 & S_{3}^{2}(u) & & \\
& S_{2}^{3}(u) & 0 & \ddots & \\
& & \ddots & & S_{r}^{r-1}(u) \\
& & & S_{r-1}^{r}(u) & S_{r}^{r}(u)
\end{array}\right)
$$

\subsection{Construction of $Q_{R}$}

As in Appendix C of [B1], we may assume that the matrix $M$ in (2.12) has the form

$$
M=\sum_{i^{\prime \prime}=1}^{r} \tilde{M}_{i^{\prime \prime}} \otimes E_{i^{\prime \prime}}^{i^{\prime \prime}}
$$

where $E_{j^{\prime \prime}}^{i^{\prime \prime}}=\left(\delta^{i^{\prime \prime}}, k^{\prime \prime} \delta_{j^{\prime \prime}, l^{\prime \prime}}\right)_{k^{\prime \prime}, l^{\prime \prime}=1, \ldots, r}$ is the $r \times r$-matrix unit and $\tilde{M}_{i^{\prime \prime}}$ is an upper triangular $2 \times 2$-matrix,

$$
\tilde{M}_{i^{\prime \prime}}=\left(\begin{array}{cc}
1 & p_{i^{\prime \prime}} \\
0 & 1
\end{array}\right)
$$

Note that the inverse of $M$ is of the following form:

$$
M^{-1}=\sum_{i^{\prime \prime}=1}^{r} \tilde{M}_{i^{\prime \prime}}^{-1} \otimes E_{i^{\prime \prime}}^{i^{\prime \prime}}
$$

The problem of constructing $S$ and $M$ satisfying (2.12) reduces to finding

- the element $p_{i^{\prime \prime}}$ of $\tilde{M}_{i^{\prime \prime}}(\underline{3.3})$ such that the $(1,2)$-element of $\tilde{M}_{i^{\prime \prime}}^{-1} L(u) \tilde{M}_{j^{\prime \prime}}$ is degenerate.

- an operator $S_{j^{\prime \prime}}^{i^{\prime \prime}}$, the image of which belongs to the kernel of the $(1,2)$ element of $\tilde{M}_{i^{\prime \prime}}^{-1} L(u) \tilde{M}_{j^{\prime \prime}}$.

Let us denote the elements of the $L$-operator as follows:

$$
L(u)=\sum_{a=0}^{3} W_{a}^{L}(u) \rho^{l}\left(S^{a}\right) \otimes \sigma^{a}=\left(\begin{array}{ll}
L_{-}^{-}(u) & L_{+}^{-}(u) \\
L_{-}^{+}(u) & L_{+}^{+}(u)
\end{array}\right) .
$$

Then, the elements of

$$
\tilde{M}_{i^{\prime \prime}}^{-1} L(u) \tilde{M}_{j^{\prime \prime}}=\left(\begin{array}{cc}
\alpha_{i^{\prime \prime}, j^{\prime \prime}}(u) & \beta_{i^{\prime \prime}, j^{\prime \prime}}(u) \\
\gamma_{i^{\prime \prime}, j^{\prime \prime}}(u) & \delta_{i^{\prime \prime}, j^{\prime \prime}}(u)
\end{array}\right)
$$


have the following form:

$$
\begin{aligned}
\alpha_{i^{\prime \prime}, j^{\prime \prime}} & =L_{-}^{-}(u)-p_{i^{\prime \prime}} L_{-}^{+}, \\
\beta_{i^{\prime \prime}, j^{\prime \prime}} & =L_{-}^{-}(u) p_{j^{\prime \prime}}+L_{+}^{-}-p_{i^{\prime \prime}} L_{-}^{+} p_{j^{\prime \prime}}-p_{i^{\prime \prime}} L_{+}^{+}, \\
\gamma_{i^{\prime \prime}, j^{\prime \prime}} & =L_{-}^{+}(u), \\
\delta_{i^{\prime \prime}, j^{\prime \prime}} & =L_{-}^{+}(u) p_{j^{\prime \prime}}+L_{+}^{+} .
\end{aligned}
$$

Substituting the expression (3.5), we have

$$
\begin{aligned}
\beta_{i^{\prime \prime}, j^{\prime \prime}}= & \left(-p_{i^{\prime \prime}}+p_{j^{\prime \prime}}\right) W_{0}(u) \rho^{l}\left(S_{0}\right)+\left(1-p_{i^{\prime \prime}} p_{j^{\prime \prime}}\right) W_{1}(u) \rho^{l}\left(S_{1}\right) \\
& +\left(-1-p_{i^{\prime \prime}} p_{j^{\prime \prime}}\right) W_{2}(u) \rho^{l}\left(S_{2}\right)+\left(p_{i^{\prime \prime}}+p_{j^{\prime \prime}}\right) W_{3}(u) \rho^{l}\left(S_{3}\right) .
\end{aligned}
$$

When we choose $p_{i^{\prime \prime}}$ and $p_{j^{\prime \prime}}$ as

$$
p_{i^{\prime \prime}}=-\frac{\theta_{00}\left(\frac{\lambda \pm 4 l \eta}{2}, \frac{\tau}{2}\right)}{\theta_{01}\left(\frac{\lambda \pm 4 l \eta}{2}, \frac{\tau}{2}\right)}, \quad p_{j^{\prime \prime}}=-\frac{\theta_{00}\left(\frac{\lambda}{2}, \frac{\tau}{2}\right)}{\theta_{01}\left(\frac{\lambda}{2}, \frac{\tau}{2}\right)}
$$

$\left(\lambda \in \mathbb{C}\right.$ is a parameter), the operator $\beta_{i^{\prime \prime}, j^{\prime \prime}}$ (3.11) is degenerate with a null vector

$$
f_{ \pm}(\lambda, u, z):=\left[z ; \frac{ \pm \lambda+u}{2}+(-l+1) \eta\right]_{2 l} \in \Theta_{00}^{4 l++}
$$

where the notation $[z ; a]_{2 l}$ is defined by (1.2). This vector is transformed by the actions of $\alpha_{i^{\prime \prime}, j^{\prime \prime}}$ and $\delta_{i^{\prime \prime}, j^{\prime \prime}}$ as follows.

$$
\begin{aligned}
& \alpha_{i^{\prime \prime}, j^{\prime \prime}} f_{ \pm}(\lambda, u, z)=\frac{\theta_{01}\left(\frac{\lambda}{2}, \frac{\tau}{2}\right)}{\theta_{01}\left(\frac{\lambda \pm 4 l \eta}{2}, \frac{\tau}{2}\right)} 2 \theta_{11}(u-2 l \eta) f_{ \pm}(\lambda, u+2 \eta, z), \\
& \delta_{i^{\prime \prime}, j^{\prime \prime}} f_{ \pm}(\lambda, u, z)=\frac{\theta_{01}\left(\frac{\lambda \pm 4 l \eta}{2}, \frac{\tau}{2}\right)}{\theta_{01}\left(\frac{\lambda}{2}, \frac{\tau}{2}\right)} 2 \theta_{11}(u+2 l \eta) f_{ \pm}(\lambda, u-2 \eta, z) .
\end{aligned}
$$

Let us set $\lambda=2\left(2 j^{\prime \prime}-1\right) l \eta$ :

$$
p_{j^{\prime \prime}}:=-\frac{\theta_{00}\left(\left(2 j^{\prime \prime}-1\right) \ln , \frac{\tau}{2}\right)}{\theta_{01}\left(\left(2 j^{\prime \prime}-1\right) \ln , \frac{\tau}{2}\right)} .
$$

Then, as we mentioned above, $\beta_{j^{\prime \prime} \pm 1, j^{\prime \prime}}$ is degenerate. In addition $\beta_{1,1}$ and $\beta_{r, r}$ are also degenerate, since $p_{0}=p_{1}$ and $p_{r}=p_{r+1}$ because of the evenness and the periodicity of theta functions. (Recall $2 l r \eta=r^{\prime} \in \mathbb{Z}$.) It is easy to check that (3.14) and (3.15) hold not only for $i^{\prime \prime}=j^{\prime \prime} \pm 1$ but also for $i^{\prime \prime}=j^{\prime \prime}=1$ and $i^{\prime \prime}=j^{\prime \prime}=r$. 
Therefore, defining an operator $S_{j^{\prime \prime}}^{i^{\prime \prime}}(u): \mathbb{C}^{r} \rightarrow \Theta_{00}^{4 l++}$ by

$$
\begin{aligned}
S_{j^{\prime \prime}}^{j^{\prime \prime} \pm 1}(u) & : \mathbb{C}^{r} \ni e_{k} \mapsto \tau_{k j^{\prime \prime}} f_{ \pm}\left(2\left(2 j^{\prime \prime}-1\right) l \eta, u, z\right) & \in \Theta_{00}^{4 l++}, \\
S_{1}^{1}(u): \mathbb{C}^{r} \ni e_{k} \mapsto \tau_{k 1} f_{-}(2 l \eta, u, z) & & \in \Theta_{00}^{4 l++}, \\
S_{r}^{r}(u): \mathbb{C}^{r} \ni e_{k} \mapsto \tau_{k r} f_{+}(2(2 r-1) l \eta, u, z) & & \in \Theta_{00}^{4 l++},
\end{aligned}
$$

$\left(\left\{e_{k}\right\}_{k=1, \ldots, r}\right.$ is the standard basis of $\left.\mathbb{C}^{r}\right)$ with generic parameters $\tau_{k j^{\prime \prime}}$ and

$$
S_{j^{\prime \prime}}^{i^{\prime \prime}}(u)=0 \text { unless } i^{\prime \prime}=j^{\prime \prime} \pm 1, i^{\prime \prime}=j^{\prime \prime}=1 \text { or } i^{\prime \prime}=j^{\prime \prime}=r,
$$

(cf. (3.1)), we obtain the operator-valued matrix $S(u)$ (2.11) satisfying (2.12).

It follows from (3.14) and (3.15) that the operators $A(u)$ and $D(u)$ in (2.12) are

$$
\begin{aligned}
& A(u)=X^{-1}(2[u-2 l \eta] S(u+2 \eta)) X \\
& D(u)=X(2[u+2 l \eta] S(u-2 \eta)) X^{-1}
\end{aligned}
$$

where $X$ is a diagonal matrix $X=\operatorname{diag}_{i^{\prime \prime}=1 \ldots, r}\left(\theta_{01}\left(\left(2 i^{\prime \prime}-1\right) l \eta, \frac{\tau}{2}\right)\right)$.

Thus the equation (2.13) reduces to the $T Q$-relation,

$$
T(u) Q_{R}(u)=h_{-}(u) Q_{R}(u-2 \eta)+h_{+}(u) Q_{R}(u+2 \eta)
$$

with the functions $h_{ \pm}(u)$ defined by (2.8).

\subsection{Hermitian conjugate and $Q_{L}$}

The operator $Q_{L}(u)$ satisfying the "QT"-relation (2.6) is constructed in a similar way as in [T4 with the help of the Sklyanin form (A.11).

The proof of the equation (3.24) in [T4] shows that the adjoint operator of the transfer matrix $T(u)$ with respect to the Sklyanin form on $\mathcal{H}$ satisfies

$$
(T(u))^{*}=(-1)^{N} T(-\bar{u}) .
$$

(When $N$ is even, this is (3.24) in [T4].) Therefore, if we define the Hermitian structure of $\mathbb{C}^{r}$ by $\left(e_{i}, e_{j}\right)=\delta_{i j}$, the adjoint of (3.20) with $-\bar{u}$ instead of $u$ gives

$$
Q_{R}(-\bar{u})^{*} T(u)=h_{+}(u) Q_{R}(-\bar{u}-2 \eta)^{*}+h_{-}(u) Q_{R}(-\bar{u}+2 \eta)^{*} .
$$

(Note that $h_{ \pm}(-\bar{u})=(-1)^{N} \overline{h_{ \pm}(u)}$.) Thus the operator $Q_{L}(u)$ defined by

$$
Q_{L}(u):=Q_{R}(-\bar{u})^{*}: \mathcal{H} \rightarrow\left(\mathbb{C}^{2 l+1}\right)^{\otimes N}
$$

satisfies the $Q T$-relation (2.6):

$$
Q_{L}(u) T(u)=h_{-}(u) Q_{L}(u-2 \eta)+h_{+}(u) Q_{L}(u+2 \eta) .
$$




\subsection{Commutation relation of $Q_{R}$ and $Q_{L}$}

Next we prove the commutation relation of $Q_{R}(u)$ and $Q_{L}(u)$,

$$
Q_{L}(u) Q_{R}\left(u^{\prime}\right)=Q_{L}\left(u^{\prime}\right) Q_{R}(u)
$$

Since the operator $Q_{R}(u)$ acts on the basis vector of $\mathbb{C}^{r}$ as

$$
Q_{R}(u) e_{i_{N}} \otimes \cdots \otimes e_{i_{1}}=\sum_{i_{1}^{\prime \prime}, \ldots, i_{N}^{\prime \prime} \in\{1, \ldots, r\}} S_{i_{N-1}^{\prime \prime}}^{i_{N}^{\prime \prime}}(u) e_{i_{N}} \otimes \cdots \otimes S_{i_{N}^{\prime \prime}}^{i_{1}^{\prime \prime}}(u) e_{i_{1}},
$$

the matrix elements of $Q_{L}(u) Q_{R}\left(u^{\prime}\right)$ has the form

$$
\begin{aligned}
& \left(e_{j_{N}} \otimes \cdots \otimes e_{j_{1}}, Q_{L}(u) Q_{R}\left(u^{\prime}\right) e_{i_{N}} \otimes \cdots \otimes e_{i_{1}}\right) \\
= & \left\langle Q_{R}(-\bar{u}) e_{j_{N}} \otimes \cdots \otimes e_{j_{1}}, Q_{R}\left(u^{\prime}\right) e_{i_{N}} \otimes \cdots \otimes e_{i_{1}}\right\rangle \\
= & \sum_{\substack{i_{1}^{\prime \prime}, \ldots, i_{N}^{\prime \prime}, j_{1}^{\prime \prime}, \ldots, j_{N}^{\prime \prime} \\
\in\{1, \ldots, r\}}} \prod_{k=1}^{N}\left\langle S_{j_{k-1}^{\prime \prime}}^{j_{k}^{\prime \prime}}(-\bar{u}) e_{j_{k}}, S_{i_{k-1}^{\prime \prime}}^{i_{k}^{\prime \prime}}\left(u^{\prime}\right) e_{i_{k}}\right\rangle \\
= & \operatorname{tr}_{\mathbb{C}^{r} \otimes \mathbb{C}^{r}} W\left(j_{N}, i_{N} \mid u, u^{\prime}\right) \cdots W\left(j_{1}, i_{1} \mid u, u^{\prime}\right),
\end{aligned}
$$

where the $r^{2} \times r^{2}$-matrix $W(j, i \mid u, v)$ is defined by

$$
W(j, i \mid u, v)=\left(\left\langle S_{j^{\prime \prime}}^{j^{\prime \prime \prime}}(u) e_{j}, S_{i^{\prime \prime}}^{i^{\prime \prime \prime}}(v) e_{i}\right\rangle\right)_{\left(j^{\prime \prime \prime}, i^{\prime \prime \prime}\right),\left(j^{\prime \prime}, i^{\prime \prime}\right) \in\{1, \ldots, r\}^{2}} .
$$

Hence, as in Appendix C of [B1] (see also [FM2]), if we can find a matrix $Y=\left(Y_{j^{\prime \prime \prime}, i^{\prime \prime \prime}}^{j^{\prime \prime}, i^{\prime \prime}}\right)_{\left(j^{\prime \prime}, i^{\prime \prime}\right),\left(j^{\prime \prime \prime}, i^{\prime \prime \prime}\right) \in\{1, \ldots, r\}^{2}}$ such that

$$
Y W(j, i \mid u, v) Y^{-1}=W(j, i \mid v, u),
$$

all the matrix elements of $Q_{L}(u) Q_{R}\left(u^{\prime}\right)$ are symmetric in $\left(u, u^{\prime}\right)$, which proves the commutation relation (3.24).

Using the results by Rosengren, [Ros1] and [Ros2] (see Appendix B), we can compute the non-zero elements of $W(j, i \mid u, v)$ explicitly:

$$
\begin{aligned}
& W(j, i \mid u, v)_{j^{\prime \prime}, i^{\prime \prime}}^{j^{\prime \prime \prime}, i^{\prime \prime \prime}}=\left\langle S_{j^{\prime \prime}}^{j^{\prime \prime \prime}}(u) e_{j}, S_{i^{\prime \prime}}^{i^{\prime \prime \prime}}(v) e_{i}\right\rangle \\
& \quad=\overline{\tau_{j j^{\prime \prime}}} \tau_{i i^{\prime \prime}} F\left(\frac{u+v}{2}+2 w_{j^{\prime \prime} i^{\prime \prime}}^{(F)^{j^{\prime \prime \prime}} i^{\prime \prime \prime}} l \eta\right) G\left(\frac{v-u}{2}+2 w^{(G)}{ }_{j^{\prime \prime} i^{\prime \prime \prime}}^{j^{\prime \prime \prime}} l \eta\right)
\end{aligned}
$$

The functions $F$ and $G$ are defined by $(\bar{B} .2)$ and $(\bar{B} .3)$ in Appendix B re-

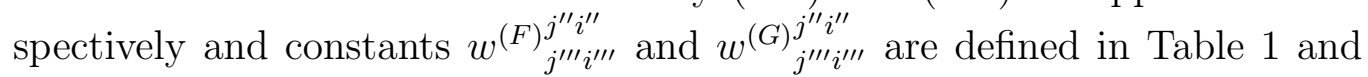




\begin{tabular}{|l||c|c|c|c|}
\hline$w^{(F)} j^{j^{\prime \prime \prime}} i^{\prime \prime \prime}$ & $j^{\prime \prime \prime}=j^{\prime \prime}+1$ & $j^{\prime \prime \prime}=j^{\prime \prime}-1$ & $j^{\prime \prime \prime}=j^{\prime \prime}=1$ & $j^{\prime \prime \prime}=j^{\prime \prime}=r$ \\
\hline \hline$i^{\prime \prime \prime}=i^{\prime \prime}+1$ & $i^{\prime \prime}-j^{\prime \prime}$ & $i^{\prime \prime}+j^{\prime \prime}-1$ & $i^{\prime \prime}$ & $i^{\prime \prime}-r$ \\
\hline$i^{\prime \prime \prime}=i^{\prime \prime}-1$ & $-i^{\prime \prime}-j^{\prime \prime}+1$ & $-i^{\prime \prime}+j^{\prime \prime}$ & $-i^{\prime \prime}+1$ & $-i^{\prime \prime}-r+1$ \\
\hline$i^{\prime \prime \prime}=i^{\prime \prime}=1$ & $-j^{\prime \prime}$ & $j^{\prime \prime}-1$ & 0 & $r$ \\
\hline$i^{\prime \prime \prime}=i^{\prime \prime}=r$ & $-j^{\prime \prime}+r$ & $j^{\prime \prime}+r-1$ & $-r$ & 0 \\
\hline
\end{tabular}

Table 1: $w^{(F)^{j^{\prime \prime \prime} i^{\prime \prime \prime}}}$ in (3.27).

\begin{tabular}{|l||c|c|c|c|}
\hline$w^{(G)^{j} j^{\prime \prime \prime} i^{\prime \prime \prime}}$ & $j^{\prime \prime \prime}=j^{\prime \prime}+1$ & $j^{\prime \prime \prime}=j^{\prime \prime}-1$ & $j^{\prime \prime \prime}=j^{\prime \prime}=1$ & $j^{\prime \prime \prime}=j^{\prime \prime}=r$ \\
\hline \hline$i^{\prime \prime \prime}=i^{\prime \prime}+1$ & $i^{\prime \prime}+j^{\prime \prime}$ & $i^{\prime \prime}-j^{\prime \prime}+1$ & $i^{\prime \prime}$ & $i^{\prime \prime}+r$ \\
\hline$i^{\prime \prime \prime}=i^{\prime \prime}-1$ & $-i^{\prime \prime}+j^{\prime \prime}+1$ & $-i^{\prime \prime}-j^{\prime \prime}+2$ & $-i^{\prime \prime}+1$ & $-i^{\prime \prime}+r+1$ \\
\hline$i^{\prime \prime \prime}=i^{\prime \prime}=1$ & $j^{\prime \prime}$ & $-j^{\prime \prime}+1$ & 0 & $r$ \\
\hline$i^{\prime \prime \prime}=i^{\prime \prime}=r$ & $j^{\prime \prime}+r$ & $-j^{\prime \prime}+r+1$ & $r$ & $2 r$ \\
\hline
\end{tabular}

Table 2: $w_{j^{\prime \prime} i^{\prime \prime}}^{(G)^{\prime \prime \prime} i^{\prime \prime \prime}}$ in (3.27).

Table 2 respectively. Except for sixteen cases in these tables, $W(j, i \mid u, v)_{j^{\prime \prime \prime}, i^{\prime \prime \prime}}^{j^{\prime \prime}, i^{\prime \prime}}$ vanishes because of the condition (3.18).

As in Appendix $\mathrm{C}$ of [B1], we take a diagonal matrix $Y$ in (3.26) of the following form:

$$
Y=\operatorname{diag}_{\left(j^{\prime \prime} i^{\prime \prime}\right) \in\{1, \ldots, r\}^{2}}\left(y_{j^{\prime \prime} i^{\prime \prime}}\right), \quad y_{j^{\prime \prime} i^{\prime \prime}}=t_{j^{\prime \prime}+i^{\prime \prime}} t_{-j^{\prime \prime}+i^{\prime \prime}+1},
$$

where $t_{m}$ is defined by the recurrence relation:

$$
\frac{t_{m+2}}{t_{m}}=\frac{G\left(\frac{u-v}{2}+2 m l \eta\right)}{G\left(\frac{v-u}{2}+2 m l \eta\right)} .
$$

We have to show (3.26), which is equivalent to

$$
\frac{y_{j^{\prime \prime \prime} i^{\prime \prime \prime}}}{y_{j^{\prime \prime} i^{\prime \prime}}}=\frac{G\left(\frac{u-v}{2}+2 w^{\left.(G)^{j^{\prime \prime \prime} i^{\prime \prime \prime}} l \eta\right)}\right.}{G\left(\frac{v-u}{2}+2 w^{(G)^{j^{\prime \prime \prime}} i^{\prime \prime \prime} i^{\prime \prime \prime} l \eta}\right)}
$$

by virtue of (3.27) and (3.28). Non-trivial cases of the left hand side of (3.30) expressed in $t_{m}$ 's are listed in Table 3 .

Comparing Table 2 and Table 3 and using properties of the function $G$, $(\mathrm{B} .4)^{2}$ and (B.5), we can show (3.30). Thus the commutation relation (3.24) has been proved.

\footnotetext{
${ }^{2}$ From the evenness (B.4) of $G$ follows $t_{m+1}=t_{-m+1}$ by induction. This property is useful when one checks (3.30).
} 


\begin{tabular}{|c|c|c|c|c|}
\hline$y_{j^{\prime \prime \prime} i^{\prime \prime \prime}} / y_{j^{\prime \prime} i^{\prime \prime}}$ & $j^{\prime \prime \prime}=j^{\prime \prime}+1$ & $j^{\prime \prime \prime}=j^{\prime \prime}-1$ & $j^{\prime \prime \prime}=j^{\prime \prime}=1$ & $j^{\prime \prime \prime}=j^{\prime \prime}=r$ \\
\hline$\overline{i^{\prime \prime \prime}=i^{\prime \prime}+1}$ & $\frac{t_{i^{\prime \prime}+j^{\prime \prime}+2}}{t_{i^{\prime \prime}+j^{\prime \prime}}}$ & $\frac{t_{i^{\prime \prime}-j^{\prime \prime}+3}}{t_{i^{\prime \prime}-j^{\prime \prime}+1}}$ & $\frac{t_{i^{\prime \prime}+2}}{t_{i^{\prime \prime}}}$ & $\frac{\bar{t}_{i^{\prime \prime}+r+1} t_{i^{\prime \prime}-r+2}}{t_{i^{\prime \prime}+r} t_{i^{\prime \prime}-r+1}}$ \\
\hline$i^{\prime \prime \prime}=i^{\prime \prime}-1$ & $\frac{t_{i^{\prime \prime}-j^{\prime \prime}-1}}{t_{i^{\prime \prime}-j^{\prime \prime}+1}}$ & $\frac{t_{i^{\prime \prime}+j^{\prime \prime}-2}}{t_{i^{\prime \prime}+j^{\prime \prime}}}$ & $\frac{t_{i_{i^{\prime \prime}-1}}}{t_{i^{\prime \prime}+1}}$ & $\frac{t_{i^{\prime \prime}+r-1} t_{i^{\prime \prime}-r}}{t_{i^{\prime \prime}+r} t_{i^{\prime \prime}-r+1}}$ \\
\hline$i^{\prime \prime \prime}=i^{\prime \prime}=1$ & $\frac{t_{j^{\prime \prime}+2} t_{-j^{\prime \prime}+1}}{t_{j^{\prime \prime}+1} t_{-j^{\prime \prime}+2}}$ & $\frac{t_{j^{\prime \prime}} t_{-j^{\prime \prime}+3}}{t_{j^{\prime \prime}+1} t_{-j^{\prime \prime}+2}}$ & 1 & 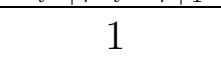 \\
\hline$i^{\prime \prime \prime}=i^{\prime \prime}=r$ & $\frac{t_{j^{\prime \prime}+r+1} t_{-j^{\prime \prime}+r}}{t_{j^{\prime \prime}+r} t_{-j^{\prime \prime}+r+1}}$ & $\frac{t_{j^{\prime \prime}+r-1} t_{-j^{\prime \prime}+r+2}}{t_{j^{\prime \prime}+r} t_{-j^{\prime \prime}+r+1}}$ & 1 & 1 \\
\hline
\end{tabular}

Table 3: $y_{j^{\prime \prime \prime} i^{\prime \prime \prime}} / y_{j^{\prime \prime} i^{\prime \prime}}$.

If there exists $u_{0} \in \mathbb{C}$ such that $Q_{R}\left(u_{0}\right)$ and $Q_{L}\left(u_{0}\right)$ are non-degenerate, multiplying $Q_{R}\left(u_{0}\right)^{-1}$ from the right and $Q_{L}\left(u_{0}\right)^{-1}$ from the left to (3.24) $\left(u^{\prime}=u_{0}\right)$, we obtain the $Q$-operator

$$
Q(u):=Q_{R}(u) Q_{R}\left(u_{0}\right)^{-1}=Q_{L}\left(u_{0}\right)^{-1} Q_{L}(u),
$$

which satisfies the $T Q$ - and $Q T$-relations, (2.5) and (2.6), because of (3.20) and (3.23). Commutativity (2.7) is a direct consequence of (3.24).

Non-degeneracy of $Q_{R}(u)$ and $Q_{L}(u)$ is quite non-trivial, as is discussed in [FM1] (see also [FM2], [FM3]), which showed that $Q_{R}(u)$ has a large kernel for certain values of $\eta$ and $N$. This problem would be discussed in a separate paper.

\subsection{Quasi-periodicity of the $Q$-operator}

The unitary operators $U_{1}$ and $U_{3}$, (A.15), act on $f_{ \pm}(\lambda, u, z)$, (3.13), as follows.

$$
\begin{aligned}
& U_{1} f_{ \pm}(\lambda, u, z)=e^{-l \pi i} f_{ \pm}(\lambda, u+1, z), \\
& U_{3} f_{ \pm}(\lambda, u, z)=e^{l(\tau-1) \pi i+2 l( \pm \lambda+u+2 l \eta) \pi i} f_{ \pm}(\lambda, u+\tau, z) .
\end{aligned}
$$

Hence, acting $U_{a}$ on (3.17), we obtain

$$
\begin{aligned}
& U_{1} S_{j^{\prime \prime}}^{i^{\prime \prime}}(u)=e^{-l \pi i} S_{j^{\prime \prime}}^{i^{\prime \prime}}(u+1), \\
& U_{3} S_{j^{\prime \prime}}^{i^{\prime \prime}}(u)=e^{l(\tau-1) \pi i+2 l \pi i u+8 d\left(i^{\prime \prime}, j^{\prime \prime}\right) l^{2} \eta \pi i} S_{j^{\prime \prime}}^{i^{\prime \prime}}(u+\tau),
\end{aligned}
$$

where

$$
d\left(i^{\prime \prime}, j^{\prime \prime}\right)= \begin{cases}j^{\prime \prime} & \left(i^{\prime \prime}=j^{\prime \prime}+1\right), \\ -i^{\prime \prime} & \left(i^{\prime \prime}=j^{\prime \prime}-1\right), \\ 0 & \left(i^{\prime \prime}=j^{\prime \prime}=1 \text { or } r\right) .\end{cases}
$$


(Recall that $8 l^{2} r \eta \in 2 \mathbb{Z}$.) The quasi-periodicity of $Q_{R}(u)$ with respect to $u \mapsto u+1$ is derived directly from (3.33), because of the definitions (2.9) and (2.10):

$$
U_{1}^{\otimes N} Q_{R}(u)=e^{-N \pi i l} Q_{R}(u+1) .
$$

In order to derive the quasi-periodicity with respect to $u \mapsto u+\tau$, a similarity transformation of $S(u)$ by a diagonal matrix

$$
S(u) \mapsto A S(u) A^{-1}, \quad A=\operatorname{diag}_{i^{\prime \prime}=1, \ldots, r}\left(A_{i^{\prime \prime}}\right), A_{i^{\prime \prime}}:=\exp \left(4 j^{\prime \prime}\left(j^{\prime \prime}-1\right) l^{2} \eta \pi i\right),
$$

is necessary. This transformation does not change $Q_{R}(u)$ thanks to $\operatorname{tr}_{\mathbb{C}^{r}}$ in (2.9) and cancels the factors in (3.33) depending on $i^{\prime \prime}$ and $j^{\prime \prime}$. Thus we obtain

$$
U_{3}^{\otimes N} Q_{R}(u)=e^{N l \pi i(\tau-1)+2 N l \pi i u} Q_{R}(u+\tau) .
$$

The quasi-periodicity of $Q_{L}(u)$ is proved by taking the Hermitian adjoint of relations (3.35) and (3.37). For any vectors $\Phi \in \mathcal{H}$ and $v \in\left(\mathbb{C}^{2 l+1}\right)^{\otimes N}$, we have

$$
\left(Q_{L}(u) U_{a}^{\otimes N} \Phi, v\right)_{\left(\mathbb{C}^{2 l+1}\right)^{\otimes N}}=\left\langle\Phi, U_{a}^{\otimes N} Q_{R}(-\bar{u}) v\right\rangle_{\mathcal{H}}
$$

by the definition (3.22) of $Q_{L}(u)$ and the unitarity of $U_{a}$.

As for the operator $U_{1}$, we have $U_{1}^{\otimes N} Q_{R}(-\bar{u})=e^{-N \pi i l} Q_{R}(-\bar{u}+1)$ because of (3.35). Note that $f_{ \pm}(\lambda, u+2, z)=f_{ \pm}(\lambda, u, z)$, which implies $Q_{R}(u+2)=$ $Q_{R}(u)$ by virtue of (2.9) and (3.17). Therefore the right hand side of (3.38) is equal to $\left\langle\Phi, e^{-N \pi i l} Q_{R}(-\bar{u}+1) v\right\rangle_{\mathcal{H}}=\left\langle\Phi, e^{-N \pi i l} Q_{R}(-\bar{u}-1) v\right\rangle_{\mathcal{H}}$. Taking the Hermitian adjoint of the operator again, we obtain $Q_{L}(u) U_{1}^{\otimes N}=e^{N \pi i l} Q_{L}(u+$ 1). From the assumption $N l \in \mathbb{Z}$ follows

$$
Q_{L}(u) U_{1}^{\otimes N}=e^{-N l \pi i} Q_{L}(u+1) .
$$

As for the operator $U_{3}$, the composition $U_{3}^{\otimes N} Q_{R}(-\bar{u})$ in (3.38) becomes $e^{N l \pi i(\tau-1)-2 N l \pi i \bar{u}} Q_{R}(-\bar{u}+\tau)$. Because $\tau$ is purely imaginary, $-\bar{u}+\tau=$ $-\overline{(u+\tau)}$. The Hermitian adjoint is, by the definition (3.22),$e^{N l \pi i(\tau-1)+2 N l \pi i+2 N l \pi i u} Q_{L}(u+$ $\tau)$. Using the assumption $N l \in \mathbb{Z}$ again, we have

$$
Q_{L}(u) U_{3}^{\otimes N}=e^{N l \pi i(\tau-1)+2 N l \pi i u} Q_{L}(u+\tau) .
$$

Since (3.35) and (3.39), (3.37) and (3.40) have pairwise the same coefficients in the right hand side, these quasi-periodicity relations for $Q_{R}(u)$ and $Q_{L}(u)$ give the quasi-periodicity of the $Q$-operator,

$$
\begin{aligned}
& U_{1}^{\otimes N} Q(u)=Q(u) U_{1}^{\otimes N}=e^{-N l \pi i} Q(u+1), \\
& U_{3}^{\otimes N} Q(u)=Q(u) U_{3}^{\otimes N}=e^{N l \pi i(\tau-1)+2 N l \pi i u} Q(u+\tau) .
\end{aligned}
$$




\section{The $Q$-operator à la Fabricius}

Here we construct the $Q$-operator of the same model in a different way, following $[\mathrm{F}]$. The strategy is almost the same as in $₫ 3$. The matrix $S(u)$ has the form

$$
S(u)=\left(\begin{array}{ccccc}
0 & S_{2}^{1}(u) & & & S_{2 r}^{1}(u) \\
S_{1}^{2}(u) & 0 & S_{3}^{2}(u) & & \\
& S_{2}^{3}(u) & 0 & \ddots & \\
& & \ddots & & S_{2 r}^{2 r-1}(u) \\
S_{1}^{2 r}(u) & & & S_{2 r-1}^{2 r}(u) & 0
\end{array}\right),
$$

instead of (3.1) $)^{3}$. Correspondingly, we use a different matrix $M$, (3.4).

Since the trace of a product of an odd number of matrices of the above form is trivially zero, we have to assume that $N$ is even.

\subsection{Construction of $Q_{R}$}

The essential idea in $\S 3$ was to make one of off-diagonal elements of $M^{-1}(L(u) \otimes$ $S(u)) M$ in (2.12) zero, which was a consequence of degeneracy of an offdiagonal element of the twisted $L$-matrix $\tilde{M}_{i^{\prime \prime}}^{-1} L(u) \tilde{M}_{j^{\prime \prime}}$ in (3.6). We used such twisting (or gauge transformation) when we applied Takhtajan-Faddeev's generalised Bethe Ansatz in [TF] to the model ([T1] and [T2]), or when we constructed the $Q$-operator by the method of Baxter's 1973 paper [B2] ([T4]).

In this section we use the matrix $M_{\lambda}(v)$ in (3.1) of [T4] (cf. also [T1], [T2] $\left.]^{4}\right)$ as the gauge transformation matrix $\tilde{M}$ of the $L$-matrix.

$$
M_{\lambda}(v):=\left(\begin{array}{rr}
-\theta_{00}\left(\frac{\lambda-v}{2}, \frac{\tau}{2}\right) & -\theta_{00}\left(\frac{\lambda+v}{2}, \frac{\tau}{2}\right) \\
\theta_{01}\left(\frac{\lambda-v}{2}, \frac{\tau}{2}\right) & \theta_{01}\left(\frac{\lambda+v}{2}, \frac{\tau}{2}\right)
\end{array}\right) .
$$

Remark 4.1. In fact, the lower triangular part of the Gauss decomposition of $M_{\lambda}(v)$ is essentially equal to the transpose of (3.3) in Baxter's method in 93 . There the conditions $p_{0}=p_{1}$ and $p_{r}=p_{r+1}$ require $v$ to be zero, which makes $M_{\lambda}(v)$ degenerate and the Gauss decomposition diverges. Nevertheless a part of this Gauss decomposition survives and gives the matrix $\tilde{M}_{i^{\prime \prime}}$ in $\$ 3.1$, (3.3).

Let us quote several formulae from the previous work [T4]. The action of the elements of the twisted $L$-matrix ((3.2) in [T4]),

$$
L_{\lambda, \lambda^{\prime}}(u ; v)=\left(\begin{array}{ll}
\alpha_{\lambda, \lambda^{\prime}}(u ; v) & \beta_{\lambda, \lambda^{\prime}}(u ; v) \\
\gamma_{\lambda, \lambda^{\prime}}(u ; v) & \delta_{\lambda, \lambda^{\prime}}(u ; v)
\end{array}\right):=M_{\lambda}(v)^{-1} L(u) M_{\lambda^{\prime}}(v),
$$

\footnotetext{
${ }^{3}$ In [F] the size of $S(u)$ is $r \times r$. In fact, for the construction of $Q_{R}$ satisfying (3.20) and $Q_{L}$ satisfying (3.23) we can use $S(u)$ of size $r \times r$ as in $[\mathrm{F}$. But when we prove the commutation relation (3.24) in 4.2 , we need $S(u)$ of size $2 r \times 2 r$.

${ }^{4}$ The normalisations in these papers are different. Here we normalise as in T4].
} 
on a vector (a function) in $\Theta_{00}^{4 l++}$ (cf. (1.2)),

$$
\omega_{\lambda}(u ; v):=\left[z ; \frac{\lambda+u-v}{2}+(-l+1) \eta\right]_{2 l},
$$

is as follows ((3.6) and (3.8) of [T4]):

$$
\begin{aligned}
\alpha_{\lambda \pm 4 l \eta, \lambda}(u ; v) \omega_{ \pm \lambda}(u ; \pm v) & =2[u+2 l \eta] \omega_{ \pm \lambda-2 \eta}(u ; \pm v) \\
\gamma_{\lambda \pm 4 l \eta, \lambda}(u ; v) \omega_{ \pm \lambda}(u ; \pm v) & =0 \\
\delta_{\lambda \pm 4 l \eta, \lambda}(u ; v) \omega_{ \pm \lambda}(u ; \pm v) & =2[u-2 l \eta] \frac{[\lambda]}{[\lambda \pm 4 l \eta]} \omega_{ \pm \lambda+2 \eta}(u ; \pm v) .
\end{aligned}
$$

Note that

$$
\begin{gathered}
M_{\lambda+4 r l \eta}(v)=M_{\lambda}(v), \\
\omega_{\lambda+4 r l \eta}(u ; v)=\omega_{\lambda}(u ; v), \\
{[\lambda+4 r l \eta]=[\lambda] .}
\end{gathered}
$$

because $2 r l \eta=r^{\prime} \in \mathbb{Z}$

Fix parameters $\lambda_{0}$ and $v$ and define

$$
\tilde{M}_{i^{\prime \prime}}:=M_{\lambda_{0}+4 i^{\prime \prime} l \eta}(v) .
$$

Then, when $i^{\prime \prime}=j^{\prime \prime} \pm 1(\bmod r)$, the matrix elements $\alpha_{i^{\prime \prime}, j^{\prime \prime}}, \gamma_{i^{\prime \prime}, j^{\prime \prime}}, \delta_{i^{\prime \prime}, j^{\prime \prime}}$ of the twisted $L$-matrix in (3.6) act on $\omega_{ \pm \lambda_{0} \pm 4 j^{\prime \prime} l \eta}(u ; \pm v)$ as follows:

$$
\begin{aligned}
\alpha_{j^{\prime \prime} \pm 1, j^{\prime \prime}}(u ; v) \omega_{ \pm \lambda_{0} \pm 4 j^{\prime \prime l} l \eta}(u ; \pm v) & =2[u+2 l \eta] \omega_{ \pm \lambda_{0} \pm 4 j^{\prime \prime l} l \eta}(u-2 \eta ; \pm v), \\
\gamma_{j^{\prime \prime} \pm 1, j^{\prime \prime}}(u ; v) \omega_{ \pm \lambda_{0} \pm 4 j^{\prime \prime} l \eta}(u ; \pm v) & =0, \\
\delta_{j^{\prime \prime} \pm 1, j^{\prime \prime}}(u ; v) \omega_{ \pm \lambda_{0} \pm 4 j^{\prime \prime l} l \eta}(u ; \pm v) & =2[u-2 l \eta] \frac{\left[\lambda_{0}+4 j^{\prime \prime} l \eta\right]}{\left[\lambda_{0}+4\left(j^{\prime \prime} \pm 1\right) l \eta\right]} \omega_{ \pm \lambda_{0} \pm 4 j^{\prime \prime} l \eta}(u+2 \eta ; \pm v) .
\end{aligned}
$$

(We used $\omega_{\lambda \pm 2 \eta}(u ; v)=\omega_{\lambda}(u \pm 2 \eta ; v)$.)

Hence, if we define $S(u)=\left(S_{j^{\prime \prime}}^{i^{\prime \prime}}(u)\right)_{i^{\prime \prime}, j^{\prime \prime}=1, \ldots, 2 r}$ by

$$
S_{j^{\prime \prime}}^{j^{\prime \prime} \pm 1(\bmod 2 r)}(u): \mathbb{C}^{r} \ni e_{k} \mapsto \tau_{k j^{\prime \prime}} \omega_{ \pm \lambda_{0} \pm 4 j^{\prime \prime l} l \eta}(u ; \pm v)
$$

$\left(\left\{e_{k}\right\}_{k=1, \ldots, 2 r}\right.$ is the standard basis of $\left.\mathbb{C}^{2 r}\right)$ and

$$
S_{j^{\prime \prime}}^{i^{\prime \prime}}(u)=0 \text { unless } i^{\prime \prime}=j^{\prime \prime} \pm 1 \quad(\bmod 2 r),
$$

(that is to say, $S(u)$ is of the form (4.1)), we have (cf. (2.12))

$$
M^{-1}(L(u) \otimes S(u)) M=\left(\begin{array}{cc}
A(u) & * \\
0 & D(u)
\end{array}\right),
$$


where $M$ is defined by

$$
M=\sum_{i^{\prime \prime}=1}^{2 r} \tilde{M}_{i^{\prime \prime}} \otimes E_{i^{\prime \prime}}^{i^{\prime \prime}}
$$

with $\tilde{M}_{i^{\prime \prime}}$ in (4.9). The matrices $A(u)$ and $D(u)$ in (4.13) are

$$
\begin{aligned}
& A(u)=2[u+2 l \eta] S(u-2 \eta), \\
& D(u)=X^{-1}(2[u-2 l \eta] S(u+2 \eta)) X,
\end{aligned}
$$

where $X=\operatorname{diag}_{i^{\prime \prime}=1, \ldots, r}\left(\left[\lambda_{0}+4 i^{\prime \prime} l \eta\right]\right)$. Therefore, as in $\oint \underline{3}$, we obtain the relation (2.13), which reduces to the $T Q$-relation (3.20) with the same coefficients $h_{ \pm}(u)$ as before.

\section{2 $Q_{L}$ and its commutation relation with $Q_{R}$}

The construction of the operator $Q_{L}$ is exactly the same as in $\$ 3.2$. Namely, the operator defined by (3.22) satisfies the $Q T$-relation (3.23).

As in 3.3 , we need to show the commutation relation of $Q_{R}(u)$ and $Q_{L}\left(u^{\prime}\right)$, (3.24). The strategy of the proof is the same as in $\$ 3.3$; Find a matrix $Y$ satisfying (3.26) for the matrix $W(j, i \mid u, v)$ defined by (3.25).

The matrix elements $W(j, i \mid u, v)_{j^{\prime \prime}, i^{\prime \prime}}^{j^{\prime \prime \prime}, i^{\prime \prime \prime}}=\left\langle S_{j^{\prime \prime}}^{j^{\prime \prime \prime}}(u) e_{j}, S_{i^{\prime \prime}}^{i^{\prime \prime \prime}}(v) e_{i}\right\rangle$ in the matrix $W(j, i \mid u, v)$ are non-zero only when $j^{\prime \prime \prime}=j^{\prime \prime} \pm 1(\bmod 2 r)$ and $i^{\prime \prime \prime}=i^{\prime \prime} \pm 1$ $(\bmod 2 r)$ and have the following form by the formula (B.6):

$$
\begin{aligned}
& W(j, i \mid u, v)_{j^{\prime \prime}, i^{\prime \prime \prime}}^{j^{\prime \prime \prime}, i^{\prime \prime \prime}} \\
= & \frac{\tau_{j j^{\prime \prime}}}{i i_{i \prime \prime}^{\prime \prime}} C_{2 l}^{\prime} \\
& \times \theta_{00}^{(2 l)}\left(\sqrt{-1} \operatorname{Im}+2\left(i^{\prime \prime}-j^{\prime \prime}\right) l \eta+\frac{1}{2} u_{j^{\prime \prime} i^{\prime \prime}}^{(1) j^{\prime \prime \prime} i^{\prime \prime \prime}}+\mu_{j^{\prime \prime} i^{\prime \prime}}^{(1) i^{\prime \prime \prime} i^{\prime \prime \prime}} l \eta\right) \\
& \times \theta_{00}^{(2 l)}\left(\operatorname{Re}+2\left(i^{\prime \prime}+j^{\prime \prime}\right) l \eta+\frac{1}{2} u_{j^{\prime \prime} i^{\prime \prime}}^{(2) i^{\prime \prime \prime}}+\mu_{j^{\prime \prime} i^{\prime \prime \prime}}^{(2) j^{\prime \prime \prime} i^{\prime \prime \prime}} l \eta\right) .
\end{aligned}
$$

(See (B.7) for notations $C_{2 l}^{\prime}$ and $\theta_{00}^{(2 l)}$.) Here, $\operatorname{Im}=\operatorname{Im}\left(\lambda_{0}-v\right)$ and $\operatorname{Re}=$ $\operatorname{Re}\left(\lambda_{0}-v\right)$. The parameters $u_{j^{\prime \prime} i^{\prime \prime}}^{(1) j^{\prime \prime \prime} i^{\prime \prime \prime}}, u_{j^{\prime \prime} i^{\prime \prime}}^{(2) j^{\prime \prime \prime} i^{\prime \prime \prime}}, \mu_{j^{\prime \prime} i^{\prime \prime}}^{(1) j^{\prime \prime \prime} i^{\prime \prime \prime}}$ and $\mu_{j^{\prime \prime} i^{\prime \prime}}^{(2) j^{\prime \prime \prime} i^{\prime \prime \prime}}$ are defined in Table 4 and Table 5 .

To find a diagonal matrix $Y=\left(Y_{j^{\prime \prime \prime}, i^{\prime \prime \prime}}^{j^{\prime \prime}, i^{\prime \prime}}\right)_{\left(j^{\prime \prime}, i^{\prime \prime}\right),\left(j^{\prime \prime \prime}, i^{\prime \prime \prime}\right) \in\{1, \ldots, r\}^{2}}$ satisfying

$$
Y W\left(j, i \mid u, u^{\prime}\right) Y^{-1}=W\left(j, i \mid u^{\prime}, u\right)
$$




\begin{tabular}{|l||c|c|}
\hline$u_{j^{\prime \prime} i^{\prime \prime}}^{(1)}, \mu_{j^{\prime \prime} i^{\prime \prime \prime}}^{(1) j^{\prime \prime \prime} i^{\prime \prime \prime}}$ & $j^{\prime \prime \prime}=j^{\prime \prime}+1$ & $j^{\prime \prime \prime}=j^{\prime \prime}-1$ \\
\hline \hline$i^{\prime \prime \prime}=i^{\prime \prime}+1$ & $u^{\prime}+u, 0$ & $u^{\prime}-u, 2$ \\
\hline$i^{\prime \prime \prime}=i^{\prime \prime}-1$ & $-u^{\prime}+u,-2$ & $-u^{\prime}-u, 0$ \\
\hline
\end{tabular}

Table 4: $u_{j^{\prime \prime} i^{\prime \prime}}^{(1) i^{\prime \prime \prime} i^{\prime \prime \prime}}$ and $\mu_{j^{\prime \prime} i^{\prime \prime}}^{(1) j^{\prime \prime \prime} i^{\prime \prime \prime}}$ in (4.16).

\begin{tabular}{|l||c|c|}
\hline$u_{j^{\prime \prime} i^{\prime \prime}}^{(2) i^{\prime \prime \prime}}, \mu_{j^{\prime \prime} i^{\prime \prime}}^{(2) j^{\prime \prime \prime} i^{\prime \prime \prime}}$ & $j^{\prime \prime \prime}=j^{\prime \prime}+1$ & $j^{\prime \prime \prime}=j^{\prime \prime}-1$ \\
\hline \hline$i^{\prime \prime \prime}=i^{\prime \prime}+1$ & $u^{\prime}-u, 2$ & $u^{\prime}+u, 0$ \\
\hline$i^{\prime \prime \prime}=i^{\prime \prime}-1$ & $-u^{\prime}-u, 0$ & $-u^{\prime}+u,-2$ \\
\hline
\end{tabular}

Table 5: $u_{j^{\prime \prime} i^{\prime \prime}}^{(2) i^{\prime \prime \prime} i^{\prime \prime \prime}}$ and $\mu_{j^{\prime \prime} i^{\prime \prime}}^{(2) j^{\prime \prime \prime} i^{\prime \prime \prime}}$ in (4.16).

we have to solve the following four series of equations:

$$
\begin{aligned}
& \frac{y_{i^{\prime \prime}+1, j^{\prime \prime}+1}}{y_{i^{\prime \prime} j^{\prime \prime}}}=\frac{\theta_{00}^{(2 l)}\left(\operatorname{Re}+2\left(i^{\prime \prime}+j^{\prime \prime}\right) l \eta+\frac{u^{\prime}-u}{2}+2 l \eta\right)}{\theta_{00}^{(2 l)}\left(\operatorname{Re}+2\left(i^{\prime \prime}+j^{\prime \prime}\right) l \eta+\frac{u-u^{\prime}}{2}+2 l \eta\right)}=: A_{i^{\prime \prime} j^{\prime \prime}}, \\
& \frac{y_{i^{\prime \prime}+1, j^{\prime \prime}-1}}{y_{i^{\prime \prime} j^{\prime \prime}}}=\frac{\theta_{00}^{(2 l)}\left(\sqrt{-1} \mathrm{Im}+2\left(i^{\prime \prime}-j^{\prime \prime}\right) l \eta+\frac{u^{\prime}-u}{2}+2 l \eta\right)}{\theta_{00}^{(2 l)}\left(\sqrt{-1} \mathrm{Im}+2\left(i^{\prime \prime}-j^{\prime \prime}\right) l \eta+\frac{u-u^{\prime}}{2}+2 l \eta\right)}=: B_{i^{\prime \prime} j^{\prime \prime}}, \\
& \frac{y_{i^{\prime \prime}-1, j^{\prime \prime}+1}}{y_{i^{\prime \prime} j^{\prime \prime}}}=\frac{\theta_{00}^{(2 l)}\left(\sqrt{-1} \mathrm{Im}+2\left(i^{\prime \prime}-j^{\prime \prime}\right) l \eta+\frac{-u^{\prime}+u}{2}-2 l \eta\right)}{\theta_{00}^{(2 l)}\left(\sqrt{-1} \mathrm{Im}+2\left(i^{\prime \prime}-j^{\prime \prime}\right) l \eta+\frac{-u+u^{\prime}}{2}-2 l \eta\right)}, \\
& \frac{y_{i^{\prime \prime}-1, j^{\prime \prime}-1}}{y_{i^{\prime \prime} j^{\prime \prime}}}=\frac{\theta_{00}^{(2 l)}\left(\operatorname{Re}+2\left(i^{\prime \prime}+j^{\prime \prime}\right) l \eta+\frac{-u^{\prime}+u}{2}-2 l \eta\right)}{\theta_{00}^{(2 l)}\left(\operatorname{Re}+2\left(i^{\prime \prime}+j^{\prime \prime}\right) l \eta+\frac{-u+u^{\prime}}{2}-2 l \eta\right)} .
\end{aligned}
$$

It is easy to show that the equation (4.21) is equivalent to (4.18) and the equation (4.20) is equivalent to (4.19). Hence what we need to solve is the system (4.18) and (4.19), namely the system of linear difference equations,

$$
\begin{aligned}
& y_{i^{\prime \prime}+1, j^{\prime \prime}+1}=A_{i^{\prime \prime} j^{\prime \prime}} y_{i^{\prime \prime} j^{\prime \prime}}, \\
& y_{i^{\prime \prime}+1, j^{\prime \prime}-1}=B_{i^{\prime \prime} j^{\prime \prime}} y_{i^{\prime \prime} j^{\prime \prime}} .
\end{aligned}
$$

The compatibility condition of this sytem is

$$
B_{i^{\prime \prime}+1, j^{\prime \prime}+1} A_{i^{\prime \prime} j^{\prime \prime}}=A_{i^{\prime \prime}+1, j^{\prime \prime}-1} B_{i^{\prime \prime} j^{\prime \prime}},
$$

which is readily checked.

We need solutions of the linear system satisfying the periodic boundary condition:

$$
y_{i^{\prime \prime}+2 r, j^{\prime \prime}}=y_{i^{\prime \prime}, j^{\prime \prime}+2 r}=y_{i^{\prime \prime} j^{\prime \prime}} .
$$


Lemma 4.2. Assume $\lambda_{0}-v=2 r^{\prime \prime} l \eta\left(r^{\prime \prime} \in \mathbb{Z}\right)$. Then for any $\left(i^{\prime \prime}, j^{\prime \prime}\right) \in \mathbb{Z}^{2}$

$$
\begin{aligned}
& \prod_{k^{\prime \prime}=0}^{r-1} A_{i^{\prime \prime}+k^{\prime \prime}, j^{\prime \prime}+k^{\prime \prime}}=1 \\
& \prod_{k^{\prime \prime}=0}^{r-1} B_{i^{\prime \prime}+k^{\prime \prime}, j^{\prime \prime}-k^{\prime \prime}}=1
\end{aligned}
$$

Hereafter we assume

$$
\lambda_{0}-v=2 r^{\prime \prime} l \eta \quad\left(r^{\prime \prime} \in \mathbb{Z}\right) .
$$

Under this assumption Lemma 4.2 shows $y_{i^{\prime \prime}+r, j^{\prime \prime}+r}=y_{i^{\prime \prime}+r, j^{\prime \prime}-r}=y_{i^{\prime \prime} j^{\prime \prime}}$ as a consequence of (4.18) and (4.19). The periodicity (4.25) directly follows from this.

Thus the existence of $Y$ satisfying (4.17) has been proved and therefore the commutation relation (3.24) has been shown.

Proof of Lemma 4.2. The proof is similar to the proof of (66) in [F].

The assumption on $\lambda_{0}-v$ means

$$
\operatorname{Re}=\operatorname{Re}\left(\lambda_{0}-v\right)=2 r^{\prime \prime} l \eta \quad \operatorname{Im}=\operatorname{Im}\left(\lambda_{0}-v\right)=0,
$$

because $\eta \in \mathbb{R}$.

By multiplying $A_{i^{\prime \prime} j^{\prime \prime}}$ 's defined by (4.18), we have

$$
\prod_{k^{\prime \prime}=0}^{r-1} A_{i^{\prime \prime}+k^{\prime \prime}, j^{\prime \prime}+k^{\prime \prime}}=\prod_{k^{\prime \prime}=0}^{r-1} \frac{\theta_{00}^{(2 l)}\left(2\left(r^{\prime \prime}+i^{\prime \prime}+j^{\prime \prime}+2 k^{\prime \prime}+1\right) l \eta+\frac{u^{\prime}-u}{2}\right)}{\theta_{00}^{(2 l)}\left(2\left(r^{\prime \prime}+i^{\prime \prime}+j^{\prime \prime}+2 k^{\prime \prime}+1\right) l \eta+\frac{u-u^{\prime}}{2}\right)} .
$$

Hence the equation (4.26) holds, if

$$
\begin{aligned}
& \left\{r^{\prime \prime}+i^{\prime \prime}+j^{\prime \prime}+2 k_{1}^{\prime \prime}+1 \quad(\bmod r) \mid k_{1}^{\prime \prime} \in\{0,1, \ldots, r-1\}\right\} \\
& \quad=\left\{-\left(r^{\prime \prime}+i^{\prime \prime}+j^{\prime \prime}+2 k_{2}^{\prime \prime}+1\right) \quad(\bmod r) \mid k_{2}^{\prime \prime} \in\{0,1, \ldots, r-1\}\right\}
\end{aligned}
$$

because $\theta_{00}^{(2 l)}(u)$ is even and has a period $2 r l \eta \in \mathbb{Z}$. The condition (4.29) means that for any $k_{1}^{\prime \prime} \in\{0,1, \ldots, r-1\}$ there exists a unique $k_{2}^{\prime \prime} \in\{0,1, \ldots, r-$ $1\}$ which satisfies

$$
r^{\prime \prime}+i^{\prime \prime}+j^{\prime \prime}+2 k_{1}^{\prime \prime}+1 \equiv-r^{\prime \prime}-i^{\prime \prime}-j^{\prime \prime}-2 k_{2}^{\prime \prime}-1 \quad(\bmod r) .
$$

It is sufficient to take $k_{2}^{\prime \prime} \in\{0,1, \ldots, r-1\}$ such that

$$
k_{2}^{\prime \prime} \equiv-\left(r^{\prime \prime}+i^{\prime \prime}+j^{\prime \prime}+k_{1}^{\prime \prime}+1\right) \quad(\bmod r),
$$


which exists uniquely. Equation (4.26) is proved.

The proof of the equation (4.27) is similar, because

$$
\prod_{k^{\prime \prime}=0}^{r-1} B_{i^{\prime \prime}+k^{\prime \prime}, j^{\prime \prime}-k^{\prime \prime}}=\prod_{k^{\prime \prime}=0}^{r-1} \frac{\theta_{00}^{(2 l)}\left(2\left(i^{\prime \prime}-j^{\prime \prime}+2 k^{\prime \prime}+1\right) l \eta+\frac{u^{\prime}-u}{2}\right)}{\theta_{00}^{(2 l)}\left(2\left(i^{\prime \prime}-j^{\prime \prime}+2 k^{\prime \prime}+1\right) l \eta+\frac{u-u^{\prime}}{2}\right)},
$$

thanks to the assumptionn $\mathrm{Im}=0$.

As in $\$ 3.3$, if there are non-degenerate $Q_{R}\left(u_{0}\right)$ and $Q_{L}\left(u_{0}\right)$, the $Q$-operator satisfying the $T Q$-relation (2.5), the $Q T$-relation (2.6) and commutativity (2.7) can be constructed by (3.31). We do not discuss the non-degeneracy problem here.

\subsection{Quasi-periodicity of the $Q$-operator}

The unitary operators $U_{a}(a=1,3)$ act on $\omega_{\lambda}(u ; v)$ defined by (4.4) as follows.

$$
\begin{aligned}
& U_{1} \omega_{\lambda}(u ; v)=e^{-l \pi i} \omega_{\lambda}(u+1, v), \\
& U_{3} \omega_{\lambda}(u, v)=e^{l(\tau-1) \pi i+2 l(\lambda+u-v+2 l \eta) \pi i} \omega_{\lambda}(u+\tau, v) .
\end{aligned}
$$

Hence we obtain

$$
\begin{aligned}
& U_{1} S_{j^{\prime \prime}}^{i^{\prime \prime}}(u)=e^{-l \pi i} S_{j^{\prime \prime}}^{i^{\prime \prime}}(u+1), \\
& U_{3} S_{j^{\prime \prime}}^{i^{\prime \prime}}(u)=e^{l(\tau-1) \pi i+2 l \pi i u+2 l \pi i d\left(i^{\prime \prime}, j^{\prime \prime}\right)} S_{j^{\prime \prime}}^{i^{\prime \prime}}(u+\tau),
\end{aligned}
$$

from (4.11). Here

$$
d\left(i^{\prime \prime}, j^{\prime \prime}\right)=\left\{\begin{array}{lll}
2\left(2 j^{\prime \prime}+1\right) l \eta+\left(\lambda_{0}-v\right) & \left(i^{\prime \prime}=j^{\prime \prime}+1\right. & (\bmod 2 r)), \\
-2\left(2 i^{\prime \prime}+1\right) l \eta-\left(\lambda_{0}-v\right) & \left(i^{\prime \prime}=j^{\prime \prime}-1\right. & (\bmod 2 r)) .
\end{array}\right.
$$

As in $\$ 3.4$, the quasi-periodicity of $Q_{R}(u)$ with respect to $u \mapsto u+1$ follows directly from (4.31):

$$
U_{1}^{\otimes N} Q_{R}(u)=e^{-N \pi i l} Q_{R}(u+1) .
$$

The quasi-periodicity with respect to $u \mapsto u+\tau$ is derived using a similarity transformation of $S(u)$ by a diagonal matrix

$$
S(u) \mapsto A S(u) A^{-1}, \quad A=\operatorname{diag}_{i^{\prime \prime}=1, \ldots, r}\left(A_{i^{\prime \prime}}\right), A_{i^{\prime \prime}}:=\exp \left(4 j^{\prime \prime}\left(j^{\prime \prime}+r^{\prime \prime}\right) l^{2} \eta \pi i\right),
$$

where $r^{\prime \prime}$ is the integer in (4.28). Note that $A_{i^{\prime \prime}+2 r}=A_{i^{\prime \prime}}$ because of the rationality $\eta=\frac{r^{\prime}}{2 r l}$. As a result of (4.31), we have

$$
U_{3}^{\otimes N} Q_{R}(u)=e^{N l \pi i(\tau-1)+2 N l \pi i u} Q_{R}(u+\tau) .
$$


(cf. (3.37).)

Derivation of the quasi-periodicity of the operator $Q_{L}$ from that of $Q_{R}$ is also the same as in $\$ 3.4$. (Note that $Q_{R}(u+2)=Q_{R}(u)$ follows from $\left.\omega_{\lambda}(u+2, v)=\omega_{\lambda}(u, v).\right)$

Thus we obtain the quasi-periodicity of the $Q$-operator

$$
\begin{aligned}
& U_{1}^{\otimes N} Q(u)=Q(u) U_{1}^{\otimes N}=e^{-N l \pi i} Q(u+1), \\
& U_{3}^{\otimes N} Q(u)=Q(u) U_{3}^{\otimes N}=e^{N l \pi i(\tau-1)+2 N l \pi i u} Q(u+\tau) .
\end{aligned}
$$

\section{Concluding remarks}

We have constructed the $Q$-operator of the higher spin eight vertex model satisfying

- $T Q$ - and $Q T$-relations, (2.5), (2.6),

- commutativity (2.7),

- quasi-periodicity, (3.41) and (4.36),

in two ways. The computations are far more complicated, but, surprisingly the strategies by Baxter and Fabricius for the spin 1/2 case (the eight vertex model) work also in higher spin cases almost as they are. This fact strongly suggests that a representation theoretical structure is hidden behind those technical complicated construction.

One clue could be the connection of Fabricius's construction and the generalised algebraic Bethe Ansatz mentioned in Remark 4.1.

Let us make several comments related to other works:

- As in our previous work [T4 the Bethe Ansatz equation for the eigenvalues of the transfer matrix and the sum rule of the Bethe roots (integrality of the sum of the Bethe roots) follow from the above mentioned properties of the $Q$-operator. We do not repeat the same derivation here.

- The $Q$-operators for the XXZ spin chain of higher spin were constructed by Roan in [Roa1, following both ways of Baxter, [B1] and [B2]. The first method is similar to the method in $\$ 4$. In the same paper other functional relation for the $Q$-operator and fused transfer matrices are derived, which should be generalised to our elliptic model. (See also [Mo for a related work.) 
- Roan's construction in [Roa2] of the $Q$-operator for the eight vertex model is similar to that by Fabricius $[\mathrm{F}]$. The main difference is the valudes of parameters. In this context our construction in this paper adds another parameter $\lambda_{0}$ by the use of the matrix $M_{\lambda}(v)$.

- The $Q$-operators for the elliptic models with infinite-dimensional state spaces were constructed by Zabrodin in [Z] and by Chicherin, Derkachov, Karakhanyan and Kirschner in [CDKK. The latter construction (especially in $\S 2$ of that paper) seems to have something in common with ours. The relation of our construction and reduction of those operators in [Z], CDKK], CDS] would be important.

- The non-locality problem mentioned in the last section of [T4] is also present in the construction of this paper, which is inevitable, if one takes Baxter's strategy. It is an interesting question whether completely different approach as in [BLZ1, [BLZ2, [Ma1, [Ma2, [BS] would be applicable.

\section{Acknowledgements}

The author expresses his gratitude to Hitoshi Konno, Kohei Motegi for discussions and encouragement and to Klaus Fabricius for informing references.

The author is grateful to Rikkyo University and Tokyo Unviersity of Marine Science and Technology for there hospitality, where parts of this work were done.

This work has been funded by the Russian Academic Excellence Project '5-100'.

\section{Appendix A Sklyanin algebra}

In this appendix we recall several facts on the Sklyanin algebra and its representations from [S1] and [S2].

The Sklyanin algebra is an associative algebra generated by four generators $S^{a}(a=0, \ldots, 3)$ subject to the following relations:

$$
L_{12}(v) L_{13}(u) R_{23}(u-v)=R_{23}(u-v) L_{13}(u) L_{12}(v) .
$$

Here the symbols are defined as follows:

- the $L$-operator $L(u)$ with a complex parameter $u$ is defined by

$$
L(u)=\sum_{a=0}^{3} W_{a}^{L}(u) S^{a} \otimes \sigma^{a},
$$


where

$$
\begin{aligned}
& W_{0}^{L}(u)=\frac{\theta_{11}(u, \tau)}{\theta_{11}(\eta, \tau)}, \quad W_{1}^{L}(u)=\frac{\theta_{10}(u, \tau)}{\theta_{10}(\eta, \tau)}, \\
& W_{2}^{L}(u)=\frac{\theta_{00}(u, \tau)}{\theta_{00}(\eta, \tau)}, \quad W_{3}^{L}(u)=\frac{\theta_{01}(u, \tau)}{\theta_{01}(\eta, \tau)} .
\end{aligned}
$$

- The matrix $R(u)$ is Baxter's R-matrix defined by

$$
R(u)=\sum_{a=0}^{3} W_{a}^{R}(u) \sigma^{a} \otimes \sigma^{a}, \quad W_{a}^{R}(u):=W_{a}^{L}(u+\eta) .
$$

Explicitly, it has the form (cf. [T2] Appendix A)

$$
R(u)=\left(\begin{array}{cccc}
a(u) & 0 & 0 & d(u) \\
0 & b(u) & c(u) & 0 \\
0 & c(u) & b(u) & 0 \\
d(u) & 0 & 0 & a(u)
\end{array}\right)
$$

where

$$
\begin{aligned}
a(u) & =C \theta_{01}(2 i t \eta, 2 i t) \theta_{01}(i t u, 2 i t) \theta_{11}(i t(u+2 \eta), 2 i t) \\
b(u) & =C \theta_{01}(2 i t \eta, 2 i t) \theta_{11}(i t u, 2 i t) \theta_{01}(i t(u+2 \eta), 2 i t) \\
c(u) & =C \theta_{11}(2 i t \eta, 2 i t) \theta_{01}(i t u, 2 i t) \theta_{01}(i t(u+2 \eta), 2 i t) \\
d(u) & =C \theta_{11}(2 i t \eta, 2 i t) \theta_{11}(i t u, 2 i t) \theta_{11}(i t(u+2 \eta), 2 i t) \\
C & =\frac{-2 e^{-\pi t u(u+2 \eta)}}{\theta_{01}(0,2 i t) \theta_{01}(2 i t \eta, 2 i t) \theta_{11}(2 i t \eta, 2 i t)}, \quad t=\frac{i}{\tau} .
\end{aligned}
$$

- The indices designate the spaces on which operators act non-trivially. For example,

$$
L_{12}(u)=\sum_{a=0}^{3} W_{a}^{L}(u) S^{a} \otimes \sigma^{a} \otimes 1, \quad R_{23}(u)=\sum_{a=0}^{3} W_{a}^{R}(u) 1 \otimes \sigma^{a} \otimes \sigma^{a} .
$$

Although the relation (A.1) contains parameters $u$ and $v$, the commutation relations among $S^{a}(a=0, \ldots, 3)$ do not depend on them:

$$
\left[S^{\alpha}, S^{0}\right]_{-}=-i J_{\alpha, \beta}\left[S^{\beta}, S^{\gamma}\right]_{+}, \quad\left[S^{\alpha}, S^{\beta}\right]_{-}=i\left[S^{0}, S^{\gamma}\right]_{+},
$$

where $(\alpha, \beta, \gamma)$ stands for an arbitrary cyclic permutation of $(1,2,3)$ and $[A, B]_{ \pm}$are the (anti-)commutator $A B \pm B A$. The structure constants $J_{\alpha, \beta}=$ $\left(\left(W_{\alpha}^{L}\right)^{2}-\left(W_{\beta}^{L}\right)^{2}\right) /\left(\left(W_{\gamma}^{L}\right)^{2}-\left(W_{0}^{L}\right)^{2}\right)$ depend on $\tau$ and $\eta$ but not on $u$. 
Let $l$ be a positive half integer. The spin $l$ representation $\rho^{l}$ of the Sklyanin algebra is defined as follows: The representation space is a space of entire functions,

$$
\begin{aligned}
\Theta_{00}^{4 l+}:=\{ & f(z) \mid \\
& \left.\quad f(z+1)=f(-z)=f(z), f(z+\tau)=\exp ^{-4 l \pi i(2 z+\tau)} f(z)\right\},
\end{aligned}
$$

which is of dimension $2 l+1$. The generator $S^{a}$ of the Sklyanin algebra acts as a difference operator on this space:

$$
\left(\rho^{l}\left(S^{a}\right) f\right)(z)=\frac{s_{a}(z-l \eta) f(z+\eta)-s_{a}(-z-l \eta) f(z-\eta)}{\theta_{11}(2 z, \tau)},
$$

where

$$
\begin{array}{ll}
s_{0}(z)=\theta_{11}(\eta, \tau) \theta_{11}(2 z, \tau), & s_{1}(z)=\theta_{10}(\eta, \tau) \theta_{10}(2 z, \tau), \\
s_{2}(z)=i \theta_{00}(\eta, \tau) \theta_{00}(2 z, \tau), & s_{3}(z)=\theta_{01}(\eta, \tau) \theta_{01}(2 z, \tau) .
\end{array}
$$

In the simplest case $l=1 / 2, \rho^{1 / 2}\left(S^{a}\right)$ are expressed by the Pauli matrices $\sigma^{a}$. We can identify $\Theta_{00}^{2+}$ and $\mathbb{C}^{2}$ by

$$
\begin{aligned}
& \theta_{00}(2 z, 2 \tau)-\theta_{10}(2 z, 2 \tau) \longleftrightarrow\left(\begin{array}{l}
1 \\
0
\end{array}\right) \\
& \theta_{00}(2 z, 2 \tau)+\theta_{10}(2 z, 2 \tau) \longleftrightarrow\left(\begin{array}{l}
0 \\
1
\end{array}\right) .
\end{aligned}
$$

Under this identification $S^{a}$ have matrix forms

$$
\rho^{1 / 2}\left(S^{a}\right)=\theta_{11}(2 \eta, \tau) \sigma^{a} .
$$

The representation space $\Theta_{00}^{4 l+}$ has a natural Hermitian structure defined by the following Sklyanin form:

$$
\langle f(z), g(z)\rangle:=\int_{0}^{1} d x \int_{0}^{\tau / i} d y \overline{f(z)} g(z) \mu(z, \bar{z}),
$$

where $z=x+i y$ and the kernel function $\mu(z, w)$ is defined by (A.12)

$$
\mu(z, w):=\frac{\theta_{11}(2 z, \tau) \theta_{11}(2 w, \tau)}{\prod_{j=0}^{2 l+1} \theta_{00}(z+w+(2 j-2 l-1) \eta, \tau) \theta_{00}(z-w+(2 j-2 l-1) \eta, \tau)} .
$$


The most important property of this sesquilinear positive definite scalar product is that the generators $S^{a}$ of the Sklyanin algebra become self-adjoint:

$$
\left(S^{a}\right)^{*}=S^{a} \text {, namely, }\left\langle f(z), S^{a} g(z)\right\rangle=\left\langle S^{a} f(z), g(z)\right\rangle .
$$

In [S2] Sklyanin also defined involutive automorphisms:

$$
X_{a}:\left(S^{0}, S^{a}, S^{b}, S^{c}\right) \mapsto\left(S^{0}, S^{a},-S^{b},-S^{c}\right),
$$

for $a=1,2,3$, where $(a, b, c)$ is a cyclic permutation of $(1,2,3)$. The unitary operators $U_{a}$ defined by

$$
\begin{aligned}
& U_{1}: \Theta_{00}^{4 \ell+} \ni f(z) \mapsto\left(U_{1} f\right)(z)=e^{\pi i \ell} f\left(z+\frac{1}{2}\right), \\
& U_{3}: \Theta_{00}^{4 \ell+} \ni f(z) \mapsto \quad\left(U_{3} f\right)(z)=e^{\pi i \ell} e^{\pi i \ell(4 z+\tau)} f\left(z+\frac{\tau}{2}\right),
\end{aligned}
$$

and $U_{2}=U_{3} U_{1}$, intertwine representations $\rho^{\ell} \circ X_{a}$ and $\rho^{\ell}: \rho^{\ell}\left(X_{a}\left(S^{b}\right)\right)=$ $U_{a}^{-1} \rho^{\ell}\left(S^{b}\right) U_{a}$. Operators $U_{a}$ satisfy the relations: $U_{a}^{2}=(-1)^{2 \ell}, U_{a} U_{b}=$ $(-1)^{2 \ell} U_{b} U_{a}=U_{c}$.

\section{Appendix B Values of Sklyanin forms of ele- ments of $\Theta_{00}^{4 l++}$}

In Appendix B of [T4] (equation (B.14)), we have computed the Sklyanin form of two shifted products of theta functions, using the results by Rosengren, [Ros1] and [Ros2] (see also Konno's work [K]):

$$
\begin{aligned}
& \left\langle[z ; \alpha]_{N},[z ; \gamma]_{N}\right\rangle \\
= & C_{N} e^{\pi i N \tau / 2} \prod_{j=0}^{N-1} \theta_{00}(\gamma-\bar{\alpha}+(2 j-N+1) \eta, \tau) \theta_{00}(\gamma+\bar{\alpha}+(2 j+N-1) \eta, \tau),
\end{aligned}
$$

where

$$
C_{N}=\frac{-2 \eta e^{3 \pi i \tau / 4}}{[2(N+1) \eta] \prod_{j=1}^{\infty}\left(1-e^{2 j \pi i \tau}\right)^{3}} .
$$

Hence, the Sklyanin form of $f_{\varepsilon}(\lambda,-\bar{u}, z)$ and $f_{\varepsilon^{\prime}}\left(\lambda^{\prime}, v, z\right)\left(\varepsilon, \varepsilon^{\prime}= \pm, \lambda, \lambda^{\prime} \in \mathbb{R}\right.$, cf. (3.13) ) has the following form:

$$
\begin{aligned}
& \left\langle f_{\varepsilon}(\lambda,-\bar{u}, z), f_{\varepsilon^{\prime}}\left(\lambda^{\prime}, v, z\right)\right\rangle \\
= & F\left(\frac{\varepsilon^{\prime} \lambda-\varepsilon \lambda}{2}+\frac{v+u}{2}\right) G\left(\frac{\varepsilon^{\prime} \lambda+\varepsilon \lambda}{2}+\frac{v-u}{2}+2(2 l-1) \eta\right),
\end{aligned}
$$


where $F$ and $G$ are defined by

$$
\begin{aligned}
& F(z):=C_{2 l} e^{\pi i l \tau} \prod_{j=0}^{2 l-1} \theta_{00}(z+(2 j-2 l+1) \eta, \tau), \\
& G(z):=\prod_{j=0}^{2 l-1} \theta_{00}(z+(2 j+2 l-1) \eta-2(2 l-1) \eta, \tau) .
\end{aligned}
$$

We shifted the argument in $G(z)$ so that $G(z)$ becomes an even function:

$$
G(-z)=G(z)
$$

It has also the periodicity:

$$
G(z+1)=G(z)
$$

because of the periodicity of $\theta_{00}$.

In $\$ 4.2$ we need the Sklyanin form among $\omega_{\lambda}(u, v)$ 's defined by (4.4). The following formula is useful.

$$
\begin{aligned}
& \left\langle\omega_{\sigma \lambda}(-\bar{u}, \sigma v), \omega_{\sigma^{\prime} \lambda^{\prime}}\left(u^{\prime}, \sigma^{\prime} v^{\prime}\right)\right\rangle \\
= & C_{2 l}^{\prime} \theta_{00}^{(2 l)}\left(\frac{\left(\lambda^{\prime}-v^{\prime}\right)-\overline{(\lambda-v)}}{2}+\frac{\sigma^{\prime} u^{\prime}+\sigma u}{2}+\left(\sigma^{\prime}-\sigma\right) l \eta\right) \\
& \times \theta_{00}^{(2 l)}\left(\frac{\left(\lambda^{\prime}-v^{\prime}\right)+\overline{(\lambda-v)}}{2}+\frac{\sigma^{\prime} u^{\prime}-\sigma u}{2}+\left(\sigma^{\prime}+\sigma\right) l \eta\right),
\end{aligned}
$$

where

$$
\begin{aligned}
C_{2 l}^{\prime} & :=C_{2 l} e^{\pi i l \tau}, \\
\theta_{00}^{(2 l)}(u) & :=\prod_{j=0}^{2 l-1} \theta_{00}(u+(2 j-2 l+1) \eta, \tau) .
\end{aligned}
$$

\section{References}

[B1] Baxter, R. J.: Partition Function of the Eight-Vertex Lattice Model, Ann. Phys. 70 (1972), 193-228.

[B2] Baxter, R. J.: Eight-Vertex Model in Lattice Statistics and OneDimensional Anisotropic Heisenberg Chain I, II, III, Ann. Phys. 76 (1973), 1-24, 25-47, 48-71. 
[B3] Baxter, R. J.: Exactly solved models in statistical mechanics. Academic Press, Inc., London, (1982), xii+486 pp.

[BLZ1] Bazhanov, V. V., Lukyanov, S. L. and Zamolodchikov, A. B.: Integrable structure of conformal field theory II, $Q$-operator and DDV equation, Comm. Math. Phys. 190 247-278 (1997)

[BLZ2] Bazhanov, V. V., Lukyanov, S. L. and Zamolodchikov, A. B.: Integrable structure of conformal field theory III, The Yang-Baxter relation, Comm. Math. Phys. 200 297-324 (1999)

[BS] Bazhanov, V. V. and Stroganov, Yu. G.: Chiral Potts model as a descendant of the six-vertex model, J. Statist. Phys. 59 799-817 (1990)

[CDKK] Chicherin, D., Derkachov, S., Karakhanyan, D. and Kirschner, R.: Baxter operators with deformed symmetry, Nuclear Phys. B 868 (2013), 652683 .

[CDS] Chicherin, D., Derkachov, S. E. and Spiridonov, V. P.: New elliptic solutions of the Yang-Baxter equation, Comm. Math. Phys. 345 (2016), 507543 .

[F] Fabricius, K.: A new Q-matrix in the eight-vertex model, J. Phys. A 40 4075-4086, (2007).

[FH] Frenkel, E., Hernandez, D.: Baxter's relations and spectra of quantum integrable models, Duke Math. J. 164, 2407-2460, (2015).

[FM1] Fabricius, K. and McCoy, B. M.: New developments in the eight vertex model, J. Statist. Phys. 111, 323-337 (2003); ditto II, Chains of odd length, J. Stat. Phys. 120 37-70, (2005).

[FM2] Fabricius, K. and McCoy, B. M.: The TQ equation of the eight-vertex model for complex elliptic roots of unity, J. Phys. A 40, 14893-14926, (2007).

[FM3] Fabricius, K. and McCoy, B. M.: New Q matrices and their functional equations for the eight vertex model at elliptic roots of unity. J. Stat. Phys. 134, 643668, (2009).

[K] Konno, H.: The vertex-face correspondence and the elliptic $6 j$ symbols, Lett. Math. Phys. 72 (2005), 243-258.

[Ma1] Mangazeev, V. V.: On the Yang-Baxter equation for the six-vertex model, Nuclear Phys. B 882 70-96 (2014) 
[Ma2] Mangazeev, V. V.: Q-operators in the six-vertex model, Nuclear Phys. B 886 166-184 (2014)

[Mo] Motegi, K.: On Baxter's Q operator of the higher spin XXZ chain at the Razumov-Stroganov point, J. Math. Phys. 54 063510, 13 pp. (2013)

[Mu] Mumford, D.: Tata Lectures on Theta I, Progress in Mathematics 28, Birkhuser Boston, Inc., (1983), xiii+235 pp.

[Roa1] Roan, S.-S.: On Q-operators of XXZ Spin Chain of Higher Spin, arXiv: cond-mat/0702271.

[Roa2] Roan, S.-S.: The Q-operator and functional relations of the eightvertex model at root-of-unity $\eta=\frac{2 m K}{N}$ for odd $N$, J. Phys. A 40, 11019-11044, (2007).

[Ros1] Rosengren, H.: Sklyanin invariant integration, Int. Math. Res. Not. 60 (2004), 3207-3232.

[Ros2] Rosengren, H.: An elementary approach to 6j-symbols (classical, quantum, rational, trigonometric, and elliptic), Ramanujan J. 13 (2007), 131-166.

[S1] Sklyanin, E. K.: Some Algebraic Structures Connected with the Yang-Baxter Equation, Funkts. analiz $i$ ego Prilozh. 16-4 (1982), 2734, (in Russian); Funct. Anal. Appl. 16 (1983), 263-270 (English transl.).

[S2] Sklyanin, E. K.: Some Algebraic Structures Connected with the Yang-Baxter Equation. Representations of Quantum Algebras, Funkts. analiz $i$ ego Prilozh. 17-4 (1983), 34-48, (in Russian); Funct. Anal. Appl. 17 (1984), 273-284, (English transl.).

[T1] Takebe, T.: Generalized Bethe Ansatz with the general spin representations of the Sklyanin algebra, J. Phys. A 25 (1992), 1071-1083.

[T2] Takebe, T.: Bethe ansatz for higher spin eight-vertex models, J. Phys. A 28 (1995), 6675-6706; Corrigendum J. Phys. A 29 (1996), 15631566 .

[T3] Takebe, T.: Bethe ansatz for higher-spin XYZ models - low-lying excitations, J. Phys. A 29 (1996), 6961-6966. 
[T4] Takebe, T.: $Q$-operators for higher spin eight vertex models with an even number of sites, Lett. Math. Phys. 106 (2016), 319340.

[TF] Takhtajan, L. A. and Faddeev, L. D.: The quantum method of the inverse problem and the Heisenberg XYZ model, Uspekhi Mat. Nauk 34:5 (1979), 13-63 (in Russian); Russian Math. Surveys 34:5 (1979), 11-68, (English translation).

[WW] Whittaker, E. T. and Watson, G. N.: A course of modern analysis, An introduction to the general theory of infinite processes and of analytic functions; with an account of the principal transcendental functions, the fourth edition, Cambridge University Press, (1927), vi+608pp.

[Z] Zabrodin, A.: Commuting difference operators with elliptic coefficients from Baxter's vacuum vectors, J. Phys. A 33 (2000), 38253850 . 